\title{
Menstrual Blood-Derived Mesenchymal Stem Cells: Women's Attitudes, Willingness, and Barriers to Donation of Menstrual Blood
}

\author{
Hannah Manley, MSc, James Sprinks, PhD, and Philip Breedon, PhD
}

\begin{abstract}
Background: Menstrual blood contains mesenchymal stem cells (MenSC), considered a potential "off-theshelf" treatment for a range of diseases and medical conditions. Samples of menstrual blood can be collected painlessly, inexpensively, and as frequently as every month for cell therapy. While there has been considerable previous research into the clinical advantages of MenSC, there is currently little understanding of potential donors' attitudes regarding menstrual blood donation and MenSC.

Methods: One hundred women 18 years of age or over were surveyed to understand attitudes and potential barriers to menstrual blood donation. The questionnaire assessed participant age and brief medical history (giving birth, donating blood, donating stem cells), menstrual experience (period rating, preferred menstrual hygiene products), and whether participants would donate MenSC or accept MenSC therapy.

Results: MenSC was met with a generally positive response, with $78 \%$ of menstruating women willing to donate menstrual blood. No significant relationship was recognized between willingness to donate menstrual blood with age, history of childbirth or blood donation, menstruation perception, and preferred menstrual hygiene product. Women rated their period experience better after being made aware of the ability to donate menstrual blood, meaning MenSC therapy can be beneficial for donors as well as patients.

Conclusions: Considering women's attitudes to MenSC and donation of menstrual blood, the future of MenSC therapy is positive; women are generally willing to donate menstrual blood, independent of age, perception of periods, and history of childbirth and blood donation.

Keywords: menstrual blood-derived mesenchymal stem cells, donation, human factors, menstrual blood, stem cells, menstruation

\section{Introduction}

$\mathbf{S}^{\mathrm{r}}$ TEM CELLS ARE cells with the ability for long-term self$\checkmark$ renewal and differentiation into multiple specialized cells. ${ }^{1}$ Embryonic stem cells are pluripotent stem cells (differentiating into all adult cell types) that would therefore appear to have huge potential for treating a huge number of diseases and conditions. However, embryonic stem cells that come with the ethical dilemma of requiring the destruction of an embryo, are not widely available, and are susceptible to teratoma formation, which is a major health risk for the embryonic stem cell transplant recipients. ${ }^{2-4}$ Adult stem cells are found across the body, and are ethically donated with consent from bone marrow, fat tissue, dental pulp, and peripheral blood. However, harvesting these cells require painful,

invasive procedures, needing specialist staff and equipment, all at a high cost. Postnatal tissues, such as placental tissue and umbilical cord blood, do not require these painful invasive procedures, and can be donated with consent, but the opportunity to donate these cells does not occur regularly. Also, early clamping and draining of the umbilical cord blood has been found to significantly lower hemoglobin levels in new borns, ${ }^{5}$ and also affects the child's neurodevelopment. ${ }^{6}$ Subsequently the World Health Organization (WHO) recognizes the dangers of early cord clamping and recommend the cord should not be clamped "earlier than necessary".

With our ever-growing, ever-aging population, the hunt for potential treatment of many more diseases and conditions continues, and the need for a safe, cheap, abundant source of stem cells grows.
\end{abstract}




\section{Menstrual blood-derived mesenchymal stem cells}

From menarche to menopause, healthy women undergo a continuous reproductive cycle in constant preparation for conception and childbirth. Single eggs are matured and released, and the uterus prepares for possible conception. If this does not happen, the woman menstruates, and the cycle starts again. Remarkably, this cyclical deterioration, shedding, and regeneration of the uterine lining occurs without the formation of any scarring: each cycle bringing new, perfectly functioning uterine tissue, suggesting stem cell-like activity. This level of controlled tissue remodeling is not found in any other organ in the body, ${ }^{8}$ and in 2004 stromal stem cells were first harvested through a hysterectomy. ${ }^{9}$

Even though 37,000 women undergo a hysterectomy each year on the NHS, ${ }^{10}$ this invasive, nonreversible procedure has the same disadvantages to the other adult stem cell sources. Also, being required by women with conditions affecting the reproductive system means a transplantation could be detrimental for the recipient.

As the menstrual cycle involves the building and shedding of the endometrium, in 2007 it was correctly postulated that menstruated blood contains mesenchymal stromal cells. ${ }^{11}$ Menstrual blood-derived mesenchymal stem cells (MenSC) are not limited in the way embryonic and other adult stem cells are: menstrual blood is a waste blood, shed from every healthy woman of reproductive age. It can be painlessly collected with every menstrual cycle, holding the potential for cell therapy that meets the growing demand on health care.

Researchers are beginning to understand the potential future applications of these cells. In animal models, MenSC have been used in the treatment of lung injury, ${ }^{12}$ spinal cord injury, ${ }^{13}$ soft tissue damage ${ }^{14}$ and critical limb ischemia, ${ }^{15}$ as well as improve cardiac function, ${ }^{16-18}$ and treatment of heart failure. ${ }^{19,20}$ Peripheral nervous system disorders have been treated, ${ }^{21}$ the outcomes of impairments after ischemic stroke have been improved with a transplantation of MenSC, ${ }^{22}$ and Alzheimer's disease-like symptoms have also been bettered. ${ }^{23}$ Premature ovarian failure, ${ }^{24}$ ovary injury as a result of cancer treatment, ${ }^{25}$ and a range of cancer types have been treated with MenSC, ${ }^{26-29}$ including allogeneic MenSC treatment in mice. ${ }^{30}$ Further success has been found in the treatment of liver failure, ${ }^{31,32}$ liver injury, ${ }^{33}$ including that from liver fibrosis, ${ }^{34}$ and liver function has been improved after sepsis. ${ }^{35}$ Finally, hyperglycemia was improved in mice with type 1 diabetes. ${ }^{36}$ In human models, with further clinical trials underway for patients with type 1 diabetes, lung damage, and liver cirrhosis, women with Asherman's syndrome received successful treatment from autologous MenSC transplants, ${ }^{37}$ and four patients with multiple sclerosis received allogeneic transplantations in four separate injections with no adverse effects and no disease progression after a 1-year follow-up, ${ }^{38}$ suggesting that future treatment could be sourced from allogeneic MenSC transplants.

With these experiments showing MenSC having the potential to treat a range of diseases and conditions, being described as potential "off-the-shelf" treatments for a range of ailments, ${ }^{3,20,36}$ it can certainly be argued that MenSC will be part of the future of medicine. There is an ever-growing understanding of the clinical outputs of MenSC, particularly in comparison to other sources of stem cells, such as bone marrow, ${ }^{31,39-44}$ or isolation, ${ }^{2,44,45}$ culture, ${ }^{18,46,47}$ and differ- entiation protocols. ${ }^{14,48}$ However, minimal work has been done to understand the attitude women (and men) have toward this therapy. Without the support of the public, and women willing to donate menstrual blood samples, there is little sense in continuing research. By speaking to the public, it will be understood whether there would be a positive reception of the potential therapy, and particularly whether the source of the cells will be morally accepted by patients. The collection method should also be discussed, as this will directly influence a woman's willingness to donate menstrual blood; without willing donors and an acceptable donation protocol, there will be insufficient quantities of MenSC for use in research and eventually therapy. With a positive reception from the public, pressure would be put on the science and medical communities to further the research in this field. These attitudes and understanding of the human factors related to the donation of menstrual blood, including the potential barriers of menstrual blood collection, were collected using a questionnaire.

Even though samples from younger donors have faster proliferation rates, ${ }^{49,50}$ MenSC have still been successfully derived and used in and ex vivo from women up to 45 years of age. ${ }^{48,51,52}$ Being such a cheap, easily accessible, and readily available source of cells for therapy, it must be argued that it is still worth collecting samples of menstrual blood from all premenopausal women when they can still be used for clinical application. Therefore, it is still important to include women from a broad age range in the survey.

\section{Materials and Methods}

\section{Participants and recruitment}

Women over the age of 18 were recruited from local universities, offices, and leisure centers to fill in a questionnaire after reading an introduction to the topic with a description of general terms. These locations were chosen to recruit participants from as broad a demographic as possible. All participants were asked to provide informed consent and were provided the opportunity to ask questions. A total number of 170 questionnaires were handed out, with 102 being returned. Two questionnaires were excluded from the study for failing to follow the questionnaire format. No incentive was provided for questionnaire completion. This research was approved by the Nottingham Trent University Ethics Clearance Subcommittee.

\section{Survey}

The questionnaire consisted of 20 questions with extra space for additional comments. Questions stemmed from five key themes: age and medical history, menstruation perception and experience, knowledge of MenSC, donation of MenSC, and receiving MenSC therapy. The brief medical history included questions such as whether the participant had given birth or donated peripheral blood in the past, and whether she menstruated. In addition to general demographic questions we also gathered information on childbirth and blood donation history. The reason for doing this is to consider the hypotheses that women with history of childbirth and therefore having been in contact with a series of bodily fluids are more comfortable donating menstrual blood. Similarly, women already happy to donate peripheral blood are happy to donate a similar, waste blood. Women who did not menstruate were not asked if they would donate their menstrual blood and the corresponding 
Table 1. Participants' Willingness to Donate Menstrual Blood Depending on Age Group, Previous Blood Donation, and Motherhood

\begin{tabular}{|c|c|c|c|}
\hline Study & $\begin{array}{l}\text { Percentage of } \\
\text { participants } \\
\text { surveyed }\end{array}$ & $\begin{array}{c}\text { Percentage } \\
\text { that would } \\
\text { donate } \\
\text { menstrual } \\
\text { blood }\end{array}$ & $\mathrm{p}$ \\
\hline Age group & & & 0.125 \\
\hline $18-24$ years & 18 & 64 & \\
\hline $25-32$ years & 28 & 84 & \\
\hline $33-40$ years & 21 & 89 & \\
\hline $41-48$ years & 16 & 70 & \\
\hline $49-55$ years & 9 & $\mathrm{n} / \mathrm{a}^{\mathrm{a}}$ & \\
\hline $56+$ years & 8 & $\mathrm{n} / \mathrm{a}^{\mathrm{a}}$ & \\
\hline $\begin{array}{l}\text { Previous blood } \\
\text { donation }\end{array}$ & & & 0.816 \\
\hline $\begin{array}{l}\text { Has previously } \\
\text { donated blood }\end{array}$ & 34 & 76 & \\
\hline $\begin{array}{l}\text { Has not } \\
\text { previously } \\
\text { donated }\end{array}$ & 66 & 79 & \\
\hline Motherhood & & & 0.520 \\
\hline Has given birth & 36 & 83 & \\
\hline $\begin{array}{l}\text { Has not given } \\
\text { birth }\end{array}$ & 64 & 76 & \\
\hline
\end{tabular}

${ }^{a}$ Figures not available as participants are no longer able to donate. ${ }^{\mathrm{b}}$ Blood donations other than menstrual blood.

questions, and they were directed straight to the last section of the questionnaire.

\section{Data analysis}

Data analysis was undertaken for quantitative components. Descriptive statistics were utilized where appropriate. Pearson chi-squared tests, and paired $t$-tests were used. $p$-Values $<0.05$ were considered significant. Open-ended, free text questions supplemented quantitative data, adding context to participants' quantitative responses.

\section{Results}

\section{Age and medical history}

A total of 100 participants completed questionnaires. Only adult women were considered (18+ years). Asking the participants for a brief medical history, $36 \%$ of participants had given birth before, $34 \%$ had donated blood before, and none of the participants had donated stem cells in the past, including donating umbilical cord blood after giving birth. Nine percent were on contraception that stops a monthly bleed, and $18 \%$ of participants did not menstruate due to being menopausal or for other health reasons, such as being severely underweight.

There was no significant relationship between age and willingness of menstruating women to donate, as shown by a Pearson chi-squared test $(\mathrm{X}(4)=7.207, p=0.125)$ (Table 1$)$. However, in women 25-32 years of age and 33-40 years of age, $84 \%$ and $89 \%$, respectively, were willing to donate their menstrual blood, showing that women of these ages are perhaps most open to the concept. There was also no significant relationship between women having given blood before and being willing to donate, as found with a Pearson chi-squared test $(\mathrm{X}(1)=0.054, p=0.816), 76 \%$ of women who had given blood would donate menstrual blood, compared with $79 \%$ of women who had not. The same was found of women who had previously given birth with their willingness to donate $(\mathrm{X}(1)=0.414, p=0.520), 83 \%$ of women who had given birth would donate menstrual blood, whereas $76 \%$ of women who had not given birth would donate.

\section{Menstruation perception and experience}

Regarding the menstruating participants' perception of their periods, they were asked to rate their experiences of menstruation on a scale 1 to 10 , from very negative to very positive, respectively. A Pearson chi-squared test showed there was no significant association between a woman's period rating and willingness to donate $(\mathrm{X}(8)=4.582, p=0.801)$. The mean (standard error) period experience score was $4.5( \pm 0.4)$, with

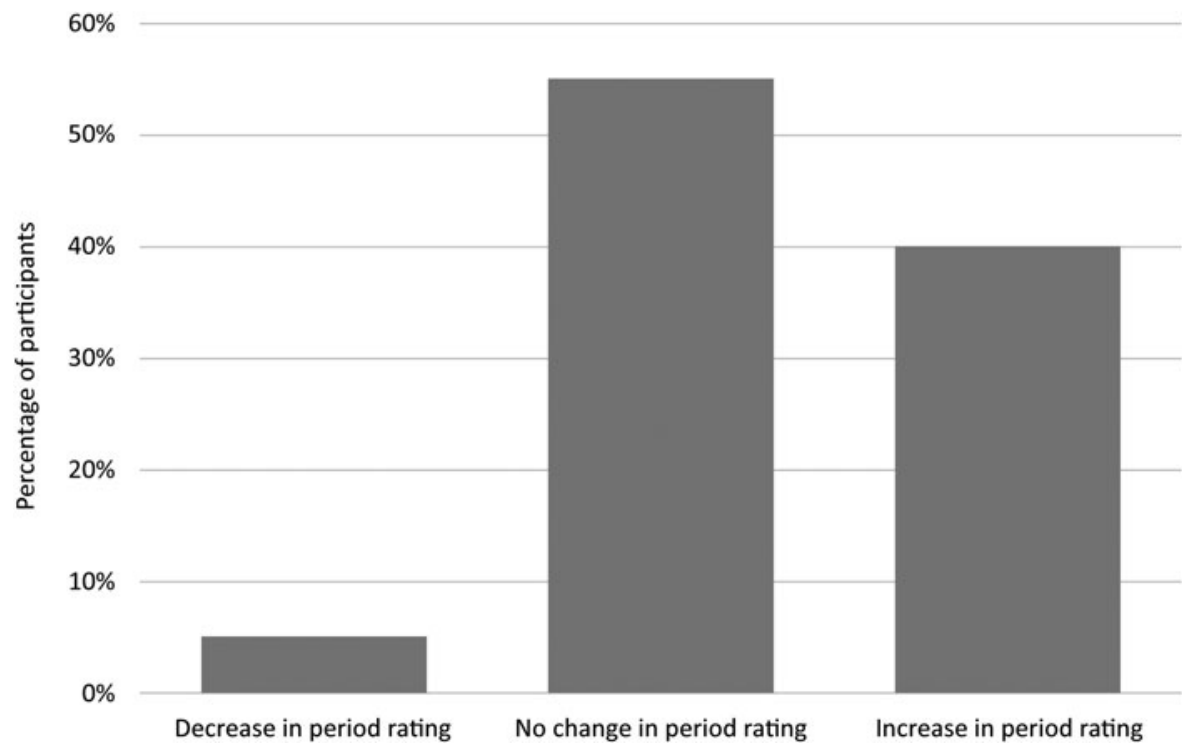

FIG. 1. Effect of knowledge of MenSC donation on participants' period experience rating. MenSC, mesenchymal stem cells. 
FIG. 2. Menstrual cup and its use.

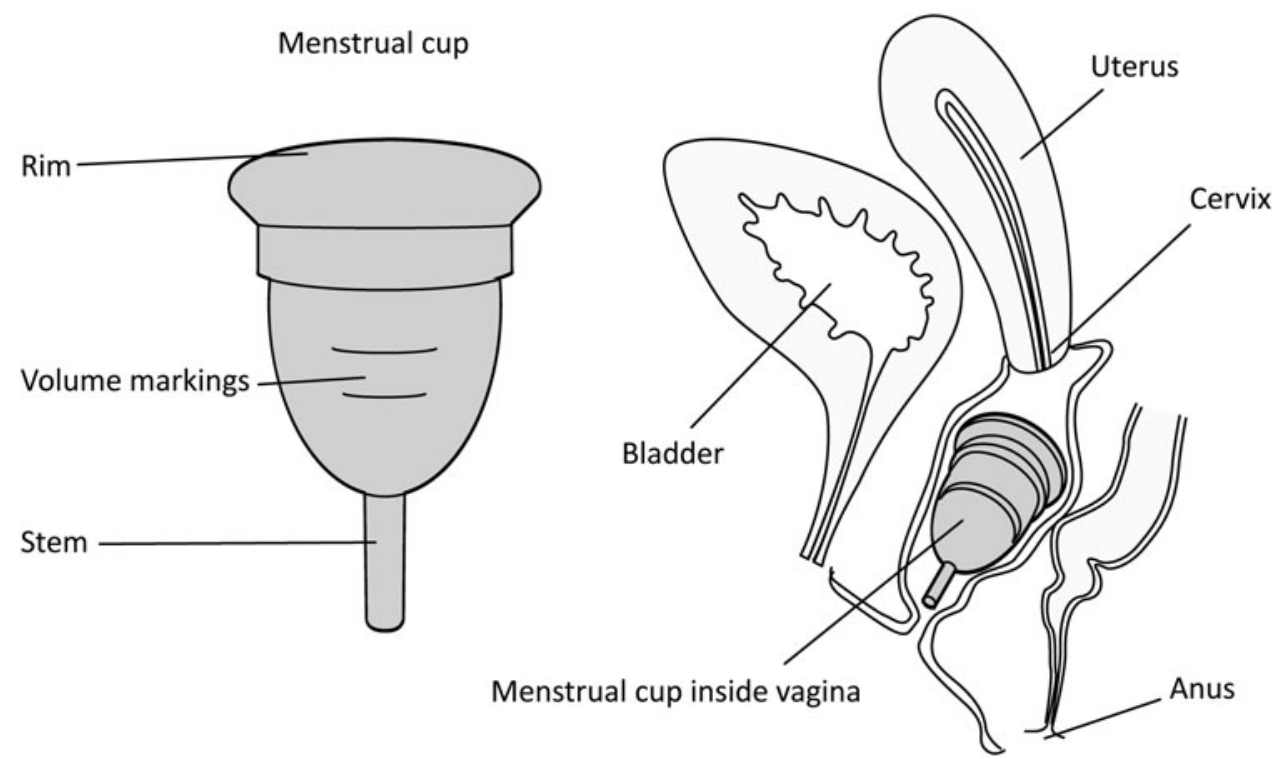

$26 \%$ of menstruating women rating their opinion on their period as positive (a score of $>5$ ), and the remaining $74 \%$ rating it as negative or neutral (a score of $\leq 5$ ). The same question was asked again, but on the pretence that the participants were able to donate MenSC. The period rating increased in $40 \%$ of cases and decreased in $5 \%$, with $55 \%$ of participants' period rating remaining unchanged (Fig. 1). A paired $t$-test shows a significant increase in participants' rating of their period $(t(69)=-4.524, p=0.001)$ after being made aware about MenSC donation (mean score $=5.6 \pm 0.4$ ).

Participants were also asked about their preferred menstrual hygiene products, because menstrual blood samples would be collected in a menstrual cup-style device, which is worn internally in the vaginal canal similarly to a tampon (Fig. 2).

When relating a menstruating woman's preferred menstrual hygiene products with their willingness to donate menstrual blood, a Pearson chi-squared test showed there was no significant association between the hygiene product groups $(\mathrm{X}(6)=2.919, p=0.819)$, with the percentage of menstruating women willing to donate ranging between $69 \%$ and $100 \%$ (Table 2). There was also no significant relationship between willingness to donate menstrual blood with preferred menstrual hygiene products worn internally, such as tampons or menstrual cups $(X(1)=0.012, p=0.914)$. Overall, $78 \%$ of women who have used an internal menstrual hygiene product were willing to donate menstrual blood, compared with $77 \%$ of women who have not.

\section{Knowledge of MenSC}

Menstruating participants were asked if they had heard of MenSC before, with $16 \%$ answering affirmatively, and of them $73 \%$ rated their opinion on MenSC as positive (a score of $\geq 6$, where 1 is very negative and 10 is very positive). The mode score was 10 , median 8 , and only $3 \%$ of participants gave a score below 5 (Fig. 3).

To add context to these scores, open-ended questions of participant thoughts on MenSC discloses themes, including menstrual blood being waste blood, so it being good to put to use, a want to develop treatment, but conversely and negatively that the idea could be "gross," or "messy" (Table 3).

\section{Donation of MenSC}

The majority of women were generally willing to donate MenSC; $78 \%$ of menstruating participants would donate a

Table 2. Participants' Willingness to Donate Menstrual Blood Depending on Preferred Menstrual Hygiene Product (InTERnal AND External)

\begin{tabular}{lcc}
\hline Study & $\begin{array}{c}\text { Percentage of } \\
\text { participants surveyed }\end{array}$ & $\begin{array}{c}\text { Percentage that } \\
\text { would donate menstrual blood }\end{array}$ \\
\hline Preferred menstrual hygiene product & 36 & 77 \\
Sanitary towel & 30 & 75 \\
Tampon & 3 & 100 \\
Menstrual cup & 22 & 69 \\
Combination: Tampons and sanitary towels & 5 & 100 \\
Combination: Menstrual cups and sanitary towels & 4 & 100 \\
Combination: Tampons and menstrual cups & & 78 \\
Type of menstrual hygiene product used: & 64 & 77 \\
Internal (i.e., tampon, menstrual cup) & 36 & 0.914 \\
External (i.e., sanitary towel) & & \\
\hline
\end{tabular}

\footnotetext{
a Participants that regularly use more than one type of menstrual hygiene product.
} 


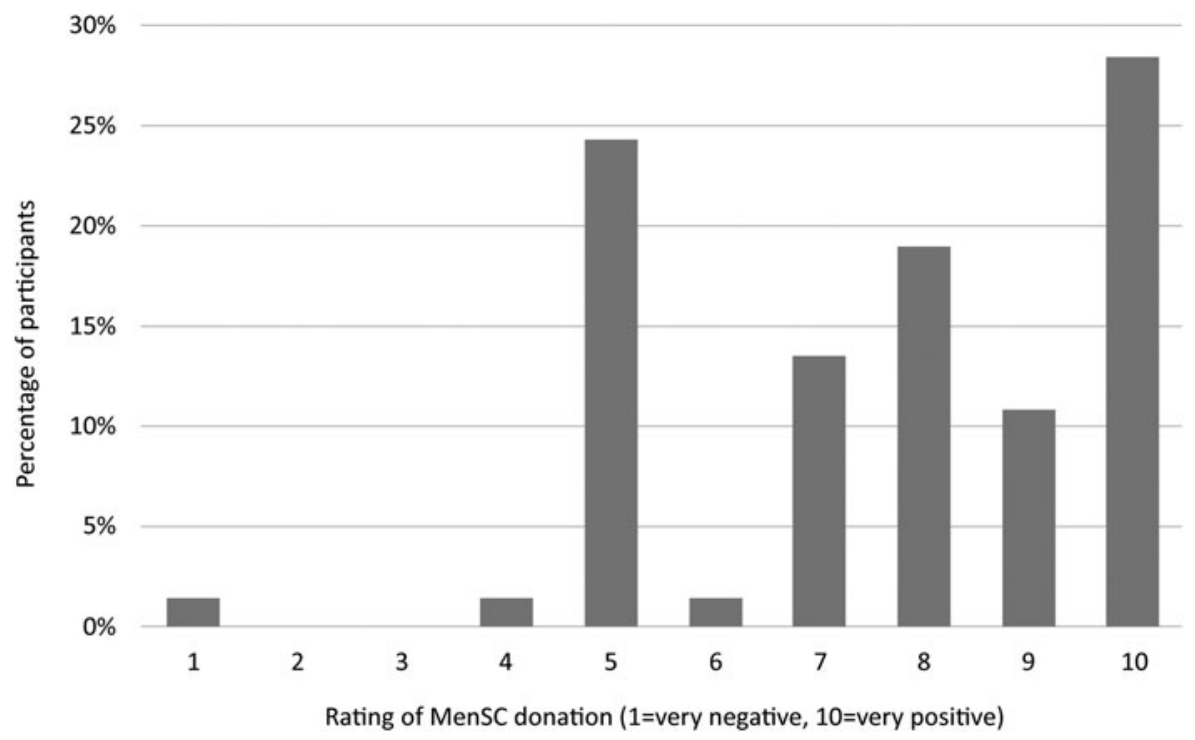

FIG. 3. Opinion of MenSC donation, for participants who have knowledge of it.

Table 3. Attitudes Toward Mesenchymal Stem Cells and Menstrual Blood Donation

\begin{tabular}{|c|c|c|}
\hline Initial question and answer & & Follow-up question \\
\hline $\begin{array}{l}\text { Had you heard before } \\
\text { today that menstrual } \\
\text { blood contains stem } \\
\text { cells? }\end{array}$ & No & $\begin{array}{l}\text { What thoughts come to } \\
\text { mind when you think } \\
\text { that menstrual blood } \\
\text { could be used in cell } \\
\text { therapy? }\end{array}$ \\
\hline
\end{tabular}

Would you donate your menstrual blood?

Yes Explain your answer
Would you use a menstrual Yes Explain your answer cup, or try using one for the first time, to donate your menstrual blood?
Participant response examples

"Menstruation affects almost $50 \%$ of the population, making it a very accessible means of cell research",

"I love it. Fantastic to think the blood I have can be used to save people's lives",

"How? What happens to the blood between collection + administration as cell therapy? Also, ew/gross/messy!",

"Seems like an excellent use of a waste product but some people might feel icky about it"

“I don't see why you wouldn't when it's just a waste product from your body it might as well be put to good use",

"If I felt I could help in any way to improve the lives of others in such a simple way it can only be a good thing",

"It seems a very easy donation to make for an important reason"

"Because I would feel disgusted maybe hygiene reasons giving where it comes from, i.e., your vagina"

"wouldn't feel confident and necessary",

" "theoretically I would, but practically I probably wouldn't for health reasons, I can't donate blood, would the same apply here?"

"I have never used a cup before but I would be willing to give it a go to help someone with stem cells that would help someone else"

"I currently use a menstrual cup, I found it empowering"

"I've heard people comment positively on this method generally. Why not kill two birds with one stone?"

"have tried cups, but didn't get on with them, tried for environmental reasons"

"I want my periods to be as unobtrusive as possible, with minimal fuss or potential for embarrassment. I imagine using a cup is less comfortable, more hassle + less convenient",

"menstrual cups are not very hygienic more likely to get toxic shock syndrome", 
Table 4. Receiving Mesenchymal Stem Cells Therapy

\begin{tabular}{|c|c|c|c|}
\hline Initial question and answer & & $\begin{array}{c}\text { Follow-up } \\
\text { question }\end{array}$ & Participant response examples \\
\hline \multirow[t]{2}{*}{$\begin{array}{l}\text { Would you accept therapy } \\
\text { from a menstrual blood } \\
\text { sample if you needed the } \\
\text { treatment? }\end{array}$} & Yes & $\begin{array}{l}\text { Explain your } \\
\text { answer }\end{array}$ & $\begin{array}{l}\text { "I don't see why using menstrual blood is any different from } \\
\text { other blood- it all comes from our bodies!" } \\
\text { "I have chronic kidney disease + high blood pressure, so if it } \\
\text { would save my life, then yes" } \\
\text { "I imagine I would be willing to try anything", }\end{array}$ \\
\hline & No & & $\begin{array}{l}\text { "Undecided would rather not have a blood transfusion" } \\
\text { "Would like to know a bit more on how you donate before } \\
\text { actually donating, depends if being on the pill affects it, } \\
\text { depends if I'm having a bad period... if it's proven to work } \\
\text { \& be hygienic then yes [would donate]" } \\
\text { "Does not feel right. Would have to think about it in more } \\
\text { detail" }\end{array}$ \\
\hline
\end{tabular}

sample of menstrual blood for cell therapy. When asked to explain their answer, of the woman willing to donate, key themes included wanting to help people and the development of medicine, menstrual blood being easy to donate, and using a waste substance. Of participants not willing to donate, fears of health affecting whether donation is possible and requiring more information prevented willingness to donate. Confidence and feelings of the donation being unhygienic were also reasons for not donating MenSC (Table 3). A Pearson chi-squared test showed a significant relationship between women willing to donate menstrual blood and those willing to use a menstrual cup to do so $(\mathrm{X}(1)=40.846, p=0.001)$. Overall, $92 \%$ of women who would donate menstrual blood would use a cup, and while this is perhaps unsurprising for those who already do so regularly, it is worth noting that $73 \%$ would use a cup for the first time to donate. Reasons given for this include having already heard positive things about menstrual cups, and the fact it would be for a beneficial purpose (Table 3). For those that would not be willing to use a menstrual cup to donate, women were adverse due to having already tried it and not got on with it, having the pre-existing thoughts that they would be uncomfortable, and the cups not being very hygienic (Table 3 ).

\section{Receiving MenSC therapy}

All women, whether they menstruated or not, were asked if they would accept cell therapy from MenSC. While 7\% of participants did not answer the question, $91 \%$ of menstruating participants said they would receive MenSC. Open-ended responses explaining participants' answers include that the source of treatment did not matter, and if help was needed, then any treatment would be welcome (Table 4). As the dependant and independent variables were categorical, a Pearson chi-squared test was used. As expected, the test showed a significant relationship between women who would donate MenSC for therapy and those accepting therapy $(\mathrm{X}(1)=7.883$, $p=0.005$ ), with $98 \%$ of women willing to donate MenSC also willing to accept MenSC therapy. Of the women who would not donate their MenSC, 77\% would still accept MenSC cell therapy. This would often be due to women having a chronic illness, as women felt they were unfit for donation for health

Table 5. Further Comments

Further comments

theme

Contraception

Blood donation

Health

Taboo

\section{Participant response examples}

"I don't currently have periods (contraception) but would be happy to use a menstrual cup to donate when I do"

"If I was to menstruate every month and it could be donated, I would do this"

"The only reason I haven't donated blood or anything before is because I am scared of the pain \& the process but I don't feel that this would be a problem with menstrual blood donation"

"And it's all good for people with a fear of needles"

"My contraception is an IUD for treatment of endometriosis. I did not choose to not menstruate, it is a side effect of my treatment. If I could be involved in something like this I would, but would have doubts about whether my hormone imbalance or treatment would affect it."

"Would the blood be ok if I'm on levothyroxine?"

"By donating menstrual blood, women may become more open in discussing their periods, something which I think is really important, rather than it being viewed as a dirty process or something to be embarrassed by",

"If menstrual blood was seen as something useful it may dispel some of the taboos around it + make young women more comfortable with the whole idea"

IUD, intrauterine device. 
reasons but would appreciate treatment. Others would feel uncomfortable donating but would still accept MenSC treatment if they were ill (Table 4). Of the women that would not accept MenSC therapy, women stated the treatment would make them feel uncomfortable, or they choose to not accept blood transfusions for personal reasons.

Further comments revealed several women wanted to reiterate their ill health and the wish to donate or receive MenSC if they were able to do it. Women who were currently on contraception that prevented menstruation stated they would donate when they stopped taking it. Donating MenSC was more appealing than donating blood or other stem cells as the donation does not require needles and would be relatively painless. Finally, the menstrual taboo was mentioned, the donation of MenSC being a potential dispelling agent of the stigma surrounding menstruation (Table 5).

\section{Limitations}

With little prior research to the topic of menstrual blood donation and attitudes toward MenSC therapy, there has been little consideration of the barriers to acceptance. History of childbirth, whole blood donation, and stem cell donation were used as key variables among age and menstruation to minimize the number of demographic questions on the questionnaire. This was to increase the number of returned questionnaires. There is an unlimited number of potential socioeconomic, cultural, and medical factors that could affect women's attitudes and willingness to donate menstrual blood or MenSC therapy. Therefore, future work can overcome this limitation by exploring other factors.

Revolving around this taboo subject, another limitation of this questionnaire is that data would be taken from women willing to do the survey, which may be women less affected by the menstrual taboo.

Physical questionnaires were distributed from one geographical region. Where participant comfort and wellbeing is paramount, physical questionnaires were less intrusive for the participants and women could choose how much they engaged with the researcher, including asking questions. Where this could limit the range of demographics within the sample, there is no reason to believe the sample is nonrepresentative of the population.

\section{Discussion}

MenSC undoubtedly is part of the future of cell therapy, ${ }^{3,14,25,47,53}$ becoming more attractive than other stem cell banks, such as umbilical cord blood. ${ }^{54}$ With this in mind, it is important that donor acceptance and barriers to uptake be fully considered. Without the acceptance and support from women, there would be no future for MenSC therapy.

As a subject, MenSC therapy and the existence of MenSC does not have a huge public awareness: $16 \%$ of women surveyed were aware of MenSC.

With a questionnaire return rate of $60 \%$, this shows that many women are prepared to talk about menstruation, cell therapy, and other sensitive topics. With popular culture beginning to show realistic depictions of menstrual blood, including TV adverts ${ }^{55}$ and film, ${ }^{56}$ and parity in medicine improving with the increase in awareness and improvement of treatment for conditions such as endometriosis, ${ }^{57}$ and urinary incontinence and pelvic organ prolapse, ${ }^{58}$ it is pos- sible that the menstrual taboo is beginning to dissipate. However, some women may have avoided participating in the survey due to this menstrual taboo.

This study highlights the overwhelmingly positive attitude the female public has to the donation of MenSC as well as using a menstrual cup to donate the menstrual blood sample, and expressing a positive response to receiving cell therapy from MenSC. Encouragingly, it appears that women of all ages are willing to donate menstrual blood, with no difference being made as to whether they had given birth. This means efforts to encourage women to donate their menstrual blood for MenSC therapy will not have to be directed to a particular demographic of women, and a large pool of women will be available for recruitment in preparation for MenSC therapy to become a reality. All ages of women, mothers and nonmothers, women with and without previous donation experience, can be supported and support each other in their MenSC donation.

Although not statistically significant, it could be argued that younger women are slightly less inclined to donate MenSC compared with women $25-40$ years of age. This could be due to less knowledge on stem cell therapy, and being generally fitter and healthier: health care not being on their minds. Educating the younger audience could be all it takes to maximize the number of women willing to donate MenSC. Data tailed off for women 41 years of age onward because biologically they were less likely to still be menstruating.

Although whether or not a woman had donated blood previously had no significant impact on her willingness to donate MenSC, several women had a fear of needles, preventing them from donating blood. Therefore, donating MenSC would be more appealing to these women, so the lack of needles could be a persuading factor utilized when recruiting potential donors.

There is no significant relationship between how a woman chooses to deal with her period (her preferred menstrual hygiene products), and her willingness to donate menstrual blood. This is a useful finding, as adverts and campaigns do not need to be targeted to women who, for example, are extremely ecoconscious and only ever use reusable menstrual hygiene products. Saying this, women who already have experience using a menstrual cup are already happy to donate menstrual blood in this way. For these women, donating MenSC would require minimal change to their monthly routine; they would not need to learn how to use a new product, which can take a multiple cycles' practice, and would be available to donate virtually immediately. However, with such a large percentage of women so willing to donate their menstrual blood they would be prepared to use an entirely new menstrual hygiene product, there is extremely promising future for the donation of menstrual blood with a menstrual cup. With the knowledge that many women could be suddenly learning how to use a new product, it is important to understand how best to support this choice. A small preliminary study showed that for women learning to use a menstrual cup for the first time, the more support and guidance she received, the better her experience, and faster the learning curve. $^{59}$ Therefore, for women who have never used a menstrual cup before, a more positive experience and consequently possibly higher returning rate can be achieved by being given a menstrual cup and supported through first use. As several women surveyed also had incorrect preexisting 
ideas surrounding menstrual cups, education on the subject, their safety, comfort, and ease of use must be highlighted for future donors.

No participants had previously donated stem cells, sourced from bone marrow, umbilical cord blood, or any other source. Therefore, no conclusion could be drawn from a woman's likeliness to donate had she previously donated stem cells.

Finally, no significant association was found between women's perception of menstruation, and their willingness to donate: women who thought their periods were "terrible" as well as women who rated their period maximally were similarly willing to donate their MenSC. Therefore, with the aim of recruiting donors of MenSC, there is no need to find women, for example, who find their periods a joyous occasion, or those who never have period cramps. Interestingly, it was found that women's rating of their period improved in $40 \%$ of respondents after being able to donate MenSC: participant comments suggest it was the idea of helping someone in need, or using a waste blood that would otherwise be thrown away that increased this experience rating, and the feelings of shame associated with menstruation being reduced. If donating MenSC can be a benefit for society as well as help individual women feel more positive about a natural bodily process, then the donation of MenSC is something to be celebrated. This must be highlighted to women when recruiting donors.

\section{Implications}

The results of this study show there is no need to urge women of specific demographics, including age, childbirth, and blood donation to donate menstrual blood, as these factors have no significant impact on women's willingness to donate. Other factors such as an increase in education, possibly in schools, could mean that more women are aware of donation and sample collection safety and hygiene. Support given to donating women could also lead to a positive donation experience and an improvement in their perception of menstruation, overcoming the menstrual taboo. Further investigation is needed regarding attitudes toward MenSC and donation requirements, to reveal potential barriers to MenSC donation and investigate how menstrual blood could be donated. Collection devices and donation systems can then be optimized for MenSC therapy needs and introduced into current health care systems.

\section{Conclusions}

As the first study of human factors surrounding MenSC and the donation of menstrual blood, there is a generally positive perception of MenSC, with $78 \%$ of menstruating women willing to donate and $91 \%$ of all women willing to accept MenSC therapy. This support of MenSC is highly positive, as the support from the public and individual future donors of menstrual blood suggests there is potential for MenSC therapy from a human factors' angle. Age, history of childbirth and donating blood, perception of menstruation, and preferred menstrual hygiene product have no significant relationship with a woman's willingness to donate, meaning that all women can be encouraged to donate. The exact donation method should also now be analyzed in terms of women's acceptance and clinical suitability.

\section{Acknowledgments}

The first author is supported by the Nottingham Trent University University External Engagement Seed Fund. Many thanks are given to the women who have taken part in the study.

\section{Author Disclosure Statement}

No competing financial interests exist.

\section{References}

1. Horwitz EM, Le Blanc K, Dominici M, et al. Clarification of the nomenclature for MSC: The International Society for Cellular Therapy position statement. Cytotherapy 2005;7: 393-395.

2. Patel AN, Park E, Kuzman M, Benetti F, Silva FJ, Allickson JG. Multipotent menstrual blood stromal stem cells: Isolation, characterization, and differentiation. Cell Transplant 2008;17:303-311.

3. Verdi J, Tan A, Shoae-Hassani A, Seifalian AM. Endometrial stem cells in regenerative medicine. J Biol Eng 2014;8:20.

4. Akhavan-Tavakoli M, Fard M, Khanjani S, et al. In vitro differentiation of menstrual blood stem cells into keratinocytes: A potential approach for management of wound healing. Biologicals 2017;48:66.

5. McDonald SJ, Middleton P. Effect of timing of umbilical cord clamping of term infants on maternal and neonatal outcomes. Cochrane Database Syst Rev 2008:CD004074.

6. Andersson O, Lindquist B, Lindgren M, Stjernqvist K, Domellöf M, Hellström-Westas L. Effect of delayed cord clamping on neurodevelopment at 4 years of age: A randomized clinical trial. JAMA Pediatr 2015;169:631-638.

7. WHO. Guideline: Delayed umbilical cord clamping for improved maternal and infant health and nutrition outcomes. Geneva: World Health Organization; 2014. https://www .ncbi.nlm.nih.gov/books/NBK310511/

8. Salamonsen LA. Current concepts of the mechanisms of menstruation: A normal process of tissue destruction. Trends Endocrinol Metab 1998;9:305-309.

9. Chan RW, Schwab KE, Gargett CE. Clonogenicity of human endometrial epithelial and stromal cells. Biol Reprod 2004; 70:1738-1750.

10. NHS. Hospital Episode Statistics: Hysterectomies. NHS Digital Leeds 2017; 9954.

11. Meng X, Ichim TE, Zhong J, et al. Endometrial regenerative cells: A novel stem cell population. J Transl Med 2007; 5:57.

12. Xiang B, Chen L, Wang X, Zhao Y, Wang Y, Xiang C. Transplantation of menstrual blood-derived mesenchymal stem cells promotes the repair of LPS-induced acute lung injury. Int J Mol Sci 2017;18:689.

13. Wu Q, Wang Q, Li Z, et al. Human menstrual bloodderived stem cells promote functional recovery in a rat spinal cord hemisection model. Cell Death Dis 2018;9:882.

14. Khanmohammadi M, Khanjani S, Edalatkhah $\mathrm{H}$, et al. Modified protocol for improvement of differentiation potential of menstrual blood-derived stem cells into adipogenic lineage. Cell Prolif 2014;47:615-623.

15. Murphy MP, Wang H, Patel AN, et al. Allogeneic endometrial regenerative cells: An" Off the shelf solution" for critical limb ischemia?. J Transl Med 2008;6:45. 
16. Jiang Z, an Wang J. Menstrual blood stem cells improved myocardial survival after rat myocardial infarction by paracrine. Heart 2012;98:E170.

17. Hida N, Nishiyama N, Miyoshi S, et al. Novel cardiac precursor-like cells from human menstrual blood-derived mesenchymal cells. Stem Cells 2008;26:1695-1704.

18. Rahimi M, Mohseni-Kouchesfehani H, Zarnani A, Mobini S, Nikoo S, Kazemnejad S. Evaluation of menstrual blood stem cells seeded in biocompatible Bombyx mori silk fibroin scaffold for cardiac tissue engineering. J Biomater Appl 2014;29:199-208.

19. Zhaocai Z, Jianan W. Menstrual blood stem cells attenuate post-infarction myocardial fibrosis via inhibiting endothelial to mesenchymal transition (ENDMT). Heart 2012;98: E36.

20. Jiang Z, Hu X, Yu H, et al. Human endometrial stem cells confer enhanced myocardial salvage and regeneration by paracrine mechanisms. J Cell Mol Med 2013;17:12471260.

21. Farzamfar S, Naseri-Nosar M, Ghanavatinejad A, Vaez A, Zarnani AH, Salehi M. Sciatic nerve regeneration by transplantation of menstrual blood-derived stem cells. Mol Biol Rep 2017;44:407-412.

22. Borlongan CV, Kaneko Y, Maki M, et al. Menstrual blood cells display stem cell-like phenotypic markers and exert neuroprotection following transplantation in experimental stroke. Stem Cells Dev 2010;19:439-452.

23. Zhao Y, Chen X, Wu Y, Wang Y, Li Y, Xiang C. Transplantation of human menstrual blood-derived mesenchymal stem cells alleviates Alzheimer's disease-like pathology in APP/PS1 transgenic mice. Front Mol Neurosci 2018;11: 140.

24. Wang Z, Huang K, Wang Y, Wang W, Bai H, Yang X. Localization of transplanted menstrual-derived stem cells in premature ovarian failure model of mice. J Xian Jiaotong Univ Med Sci 2017;38:803.

25. Lai D, Wang F, Yao X, Zhang Q, Wu X, Xiang C. Human endometrial mesenchymal stem cells restore ovarian function through improving the renewal of germline stem cells in a mouse model of premature ovarian failure. J Transl Med 2015;13:155.

26. Moreno R, Rojas L, Villellas FV, et al. Human menstrual blood-derived mesenchymal stem cells as potential cell carriers for oncolytic adenovirus. Stem Cells Int 2017; 2017:3615729.

27. Wang X, Xiang B, Ding Y, et al. Human menstrual bloodderived mesenchymal stem cells as a cellular vehicle for malignant glioma gene therapy. Oncotarget 2017;8: 58309.

28. Bu S, Wang Q, Zhang Q, et al. Human endometrial mesenchymal stem cells exhibit intrinsic anti-tumor properties on human epithelial ovarian cancer cells. Sci Rep 2016;6: 37019.

29. Alcayaga-Miranda F, Gonzalez PL, Lopez-Verrilli A, et al. Prostate tumor-induced angiogenesis is blocked by exosomes derived from menstrual stem cells through the inhibition of reactive oxygen species. Oncotarget 2016;7: 44462-44477.

30. Moreno R, Fajardo CA, Farrera-Sal M, et al. Enhanced antitumor efficacy of oncolytic adenovirus-loaded menstrual blood-derived mesenchymal stem cells in combination with peripheral blood mononuclear cells. Mol Cancer Ther 2019;18:127-138.
31. Chen L, Xiang B, Wang X, Xiang C. Exosomes derived from human menstrual blood-derived stem cells alleviate fulminant hepatic failure. Stem Cell Res Ther 2017;8:9.

32. Fathi-Kazerooni M, Tavoosidana G, Taghizadeh-Jahed M, et al. Comparative restoration of acute liver failure by menstrual blood stem cells compared with bone marrow stem cells in mice model. Cytotherapy 2017;19:1474-1490.

33. Mou X, Lin J, Chen J, et al. Menstrual blood-derived mesenchymal stem cells differentiate into functional hepatocytelike cells. J Zhejiang Univ Sci B 2013;14:961-972.

34. Chen L, Zhang C, Chen L, et al. Human menstrual bloodderived stem cells ameliorate liver fibrosis in mice by targeting hepatic stellate cells via paracrine mediators. Stem Cells Transl Med 2017;6:272-284.

35. Alcayaga-Miranda F, Cuenca J, Martin A, Contreras L, Figueroa FE, Khoury M. Combination therapy of menstrual derived mesenchymal stem cells and antibiotics ameliorates survival in sepsis. Stem Cell Res Ther 2015;6:199.

36. $\mathrm{Wu} \mathrm{X}$, Luo Y, Chen J, et al. Transplantation of human menstrual blood progenitor cells improves hyperglycemia by promoting endogenous progenitor differentiation in type 1 diabetic mice. Stem Cells Dev 2014;23:1245-1257.

37. Tan J, Li P, Wang Q, et al. Autologous menstrual bloodderived stromal cells transplantation for severe Asherman's syndrome. Hum Reprod 2016;31:2723-2729.

38. Zhong Z, Patel AN, Ichim TE, et al. Feasibility investigation of allogeneic endometrial regenerative cells. J Transl Med 2009;7:15.

39. Kozhukharova I, Zemelko V, Kovaleva Z, Alekseenko L, Lyublinskaya O, Nikolsky N. Therapeutic doses of doxorubicin induce premature senescence of human mesenchymal stem cells derived from menstrual blood, bone marrow and adipose tissue. Int J Hematol 2018;107:286-296.

40. Khanjani S, Khanmohammadi M, Zarnani A, et al. Comparative evaluation of differentiation potential of menstrual blood-versus bone marrow-derived stem cells into hepatocytelike cells. PLoS One 2014;9:e86075.

41. Darzi S, Zarnani AH, Jeddi-Tehrani M, et al. Osteogenic differentiation of stem cells derived from menstrual blood versus bone marrow in the presence of human platelet releasate. Tissue Eng Part A 2012;18:1720-1728.

42. Ren H, Sang Y, Zhang F, Liu Z, Qi N, Chen Y. Comparative analysis of human mesenchymal stem cells from umbilical cord, dental pulp, and menstrual blood as sources for cell therapy. Stem Cells Int 2016;2016:3516574.

43. Wang H, Jin P, Sabatino M, et al. Comparison of endometrial regenerative cells and bone marrow stromal cells. J Transl Med 2012;10:207.

44. Khanmohammadi M, Khanjani S, Bakhtyari MS, et al. Proliferation and chondrogenic differentiation potential of menstrual blood-and bone marrow-derived stem cells in two-dimensional culture. Int J Hematol 2012;95:484-493.

45. Dalirfardouei R, Jamialahmadi K, Mahdipour E. A feasible method for the isolation of mesenchymal stem cells from menstrual blood and their exosomes. Tissue Cell 2018;55: 53-62.

46. Jiang YS, Zhu HY, Jin XY, et al. Co-culture with endometrial stromal cells promotes the differentiation of menstrual blood-derived mesenchymal stem cells into endometrial epithelial cells. Zhonghua Yi Xue Za Zhi 2017;97:2614-2619.

47. Rajabi Z, Yazdekhasti H, Mugahi, Seyed Mohammad Hossein Noori, et al. Mouse preantral follicle growth in 3D 
co-culture system using human menstrual blood mesenchymal stem cell. Reprod Biol 2018;18:122-131.

48. Kazemnejad S, Zarnani A, Khanmohammadi M, Mobini S. Chondrogenic differentiation of menstrual blood-derived stem cells on nanofibrous scaffolds. In: Turksen K. Stem cell nanotechnology. Humana Press, Totowa, NJ: Springer, 2013:149-169.

49. Liu Y, Niu R, Yang F, et al. Biological characteristics of human menstrual blood-derived endometrial stem cells. J Cell Mol Med 2018;22:1627-1639.

50. Musina RA, Belyavski AV, Tarusova OV, Solovyova EV, Sukhikh GT. Endometrial mesenchymal stem cells isolated from the menstrual blood. Bull Exp Biol Med 2008;145: 539-543.

51. Nikoo S, Ebtekar M, Jeddi-Tehrani M, et al. Effect of menstrual blood-derived stromal stem cells on proliferative capacity of peripheral blood mononuclear cells in allogeneic mixed lymphocyte reaction. J Obstet Gynaecol Res 2012;38:804-809.

52. Luz-Crawford P, Torres MJ, Noël D, et al. The immunosuppressive signature of menstrual blood mesenchymal stem cells entails opposite effects on experimental arthritis and graft versus host diseases. Stem Cells 2016;34:456-469.

53. Rodrigues MCO, Voltarelli J, Sanberg PR, et al. Recent progress in cell therapy for basal ganglia disorders with emphasis on menstrual blood transplantation in stroke. Neurosci Biobehav Rev 2012;36:177-190.

54. Rodrigues MC, Glover LE, Weinbren N, et al. Toward personalized cell therapies: Autologous menstrual blood cells for stroke. J Biomed Biotechnol 2011;2011:194720.
55. Bodyform. Periods are normal, showing them should be too. 2017. [online]. https://www.bodyform.co.uk/our-world/ bloodnormal/ Accessed December 12, 2017.

56. Mary Queen of Scots [Film]. Focus Features LLC, 2018.

57. National Guideline Alliance (UK). Endometriosis: diagnosis and management. London: National Institute for Health and Care Excellence (UK); 2017 Sep. (NICE guideline, No. 73.) Available from: https://www.ncbi.nlm .nih.gov/books/NBK453273/ Accessed August 4, 2019.

58. Willet K, McLean K. Letter to Regional Directors, Trust Medical Directors, and clinicians involved in the care of patients with stress urinary incontinence and pelvic organ prolapse. 2018 [online] https://i.emlfiles $4 . c o m / c m p d o c / 9 / 7 /$ 2/8/1/1/files/47908_follow-up-mesh-letter-and-guidance-20july.pdf?utm_campaign=2146932_Vaginal\%20mesh\%20\%20follow\%20up\&utm_medium=email\&utm_source= Monitor\&utm_orgtype $=\& \mathrm{dm} \_\mathrm{t}=0,0,0,0,0 ;$ Accessed July 20, 2018.

59. Manley $H$. The potential for menstrually-Derived stem cell banking in the UK. J Undergrad Res NTU 2018;1: $1-18$.

Address correspondence to: Philip Breedon, PhD Medical Engineering Design Research Group Nottingham Trent University Nottingham NG1 4FQ United Kingdom

E-mail: philip.breedon@ntu.ac.uk 